\title{
Diversity Combining of Signals with Different Modulation Levels in Cooperative Relay Networks ${ }^{1}$
}

\author{
Akram Bin Sediq and Halim Yanikomeroglu \\ Broadband Communications and Wireless Systems (BCWS) Centre, \\ Department of Systems and Computer Engineering, Carleton University, Ottawa, Ontario K1S 5B6, Canada \\ e-mail: \{akram, halim\}@sce.carleton.ca
}

\begin{abstract}
In digital cooperative relaying, signals from the source-destination and relay-destination links are combined at the destination to achieve spatial diversity. These signals do not necessarily belong to the same modulation scheme due to the varying channel qualities of the two links. In this paper, we present novel and low complexity schemes for diversity combining of signals with different modulation levels. We start by developing the optimum solution as a maximum likelihood detector (MLD). Due to its high complexity, we propose two other receiver structures that we refer to as soft-bit maximum likelihood detector (SBMLD) and soft-bit maximum ratio combiner (SBMRC). The proposed schemes are simple bit-by-bit detectors and only $0.3 \mathrm{~dB}$ inferior to the MLD in performance. The SBMLD provides only marginal performance gain over SBMRC through the computation of the conditional probability density functions of the soft-bits. Consequently, the SBMRC is a more attractive and practical solution. The performance of SBMRC is compared to that of selection combining which is the current approach in the literature for combining signals with different modulations. The SBMRC, along with its simplicity, outperforms selection combining by almost $2 \mathrm{~dB}$ without bandwidth loss or the need for extra channel state information. The SBMRC scheme can be viewed as a more general form of the classical maximum ratio combiner (MRC).
\end{abstract}

\section{INTRODUCTION}

Next generation wireless networks require high data rates to meet the demand of newly emerged applications. The use of relays in the wireless network was shown to be a key technology to achieve these requirements [1]. Relays can be classified into digital and analogy relays. Analog relays amplify and forward (AF) the received signal while digital relays decode and forward (DF) a regenerated version of the received signal. In this work, digital relaying is considered as it is the focus of most of the next generation wireless networks standards such as IEEE 802.16j [2].

Two key strategies proposed for increasing the data rate in relay networks are cooperative relaying [3][4], and adaptive modulation and coding (AMC). While the former is used to improve the quality of the links by combining the signals received from the base station (BS) and the relay station(s), the latter is used to optimize the transmission rate according to the channel conditions. In [5] and [6], it is shown that the average throughput of the wireless network can be significantly increased by combining the two strategies. When AMC is utilized, the signals reaching the user terminal (UT) from BS and relay station (RS) don't necessarily belong to the same modulation, yet they contain the same information bits. In order to achieve spatial diversity for signals with different modulation levels, selection combining, rather than maximum ratio combining (MRC), was utilized because it was not known yet how to do MRC for signals with different modulation levels [5][6]. To the best of the authors' knowledge, the optimal technique of combining signals with different modulation schemes is not investigated yet, and this is the gap that this paper aspires to fill.

The need for such a technique arises from the nature of relay networks that impose different channel conditions on different links. For example, since the relays are fixed and can be installed at strategic locations, reliable line-of-sight (LOS) link(s) can be established between BS and RS, and hence, larger constellations can be used to achieve high data rates. However, because of the mobility of the users, the link(s) from RS to UT are not necessary as reliable, so smaller constellations can be used to ensure reliable transmission. In order to achieve spatial diversity at UT, it is imperative to establish an optimal diversity combining scheme for different modulations. The main focus of the paper will be on fixedrelay networks. Nevertheless, the extension to the case of nomadic or mobile relays is straight forward.

The rest of the paper is organized as follows. In Section II, the system model is presented. The optimal detector is developed in Section III. Section IV explains the proposed schemes. Simulation results of the proposed schemes are presented in Section V followed by conclusions in Section VI.

Notations: $\quad \mathcal{C N}\left(0, \sigma^{2}\right)$ represents a circular symmetric complex Gaussian random variable with zero mean and variance $\sigma^{2}$; for a random variable $X, \bar{X}=E\{X\}$ denotes its mean; $f_{X \mid y=c}(x)$ is the conditional probability density function of $X$ evaluated at $x$ given that $y=c$; for a complex number $C$, $\operatorname{Re}\{C\}$ and $\operatorname{Im}\{C\}$ denote the real and imaginary parts of $C$, respectively; $C^{*}$ is the complex conjugate of $C ; g\left(x ; \mu, \sigma^{2}\right)$ denotes the Gaussian probability density function (PDF) with mean $\mu$ and variance $\sigma^{2}$, i.e., $g\left(x ; \mu, \sigma^{2}\right)=(1 / \sigma \sqrt{2 \pi}) \exp \left(-(x-\mu)^{2} /\left(2 \sigma^{2}\right)\right)$.

\footnotetext{
${ }^{1}$ This work is supported by an Ontario Graduate Scholarship (OGS). A concise version of this work was presented in the Wireless World Research Forum Meeting 20 (WWRF20).
} 


\section{SYSTEM MODEL}

We consider a multihop network of $L$ transmitting nodes, (comprising of $L-1 \mathrm{RSs}$ and a BS), and a receiving UT, all having a single antenna. This layout is shown in Fig. 1. The RSs are used to assist a UT at the cell-edge or otherwise suffering from poor channel conditions. The RSs decode the signals they receive from BS and forward them to the UT.

The transmitting nodes transmit on $L$ orthogonal channels, i.e., they do not interfere with each other. For simplicity, we consider time division multiple access (TDMA) to insure orthogonal transmission from all the nodes. The transmitting node $i$, (BS or $\left.\mathrm{RS}_{\mathrm{i}}\right)$ where $i \in\{0,1, \ldots, L-1\}$, uses $M_{i}$ Quadrature Amplitude Modulation ( $\left.M_{i}-\mathrm{QAM}\right)$ with Gray coding. Each $M_{i}-$ QAM symbol carries $K_{i}$ bits, where $K_{i}=\log _{2}\left(M_{i}\right)$ and $M_{i}$ is the $i^{\text {th }}$ modulation level. Without loss of generality, the $M_{i}-$ QAM constellation has an average energy per bit equal to unity.

The frame is divided into $L$ sub-frames, i.e. one sub-frame for each transmitting node. All sub-frames contain the same sequence of $C$ bits, denoted by $\left\{s_{1}, s_{2}, \ldots, s_{C}\right\}$. The $i^{\text {th }}$ sub-frame consists of $T_{i} M_{i}-\mathrm{QAM}$ symbols, each denoted by $S_{i, j}^{M_{i}}$, where $j \in\left\{0,1, \ldots, T_{i}-1\right\}$. The symbol $S_{i, j}^{M_{i}}$ contains the bit sequence $\left\{s_{j K i+1}, s_{j K i+2}, \ldots, s_{(j+1) K i}\right\}$. Note that different nodes will be assigned different number of symbols, depending on their modulation schemes, i.e. $T_{i}=C / K_{i}$. Since $T_{i}$ is an integer, $C$ must be a common multiple of $\left\{K_{0}, K_{1}, \ldots, K_{L-1}\right\}$. Without loss of generality, $C$ will be used as the Least Common Multiple (LCM) of $\left\{K_{0}, K_{1}, \ldots, K_{L-1}\right\}$.

In the first sub-frame, BS broadcasts $T_{0} M_{0}-\mathrm{QAM}$ symbols to all RSs and the UT. Since the RSs can be installed at strategic locations, LOS transmission between BS and the RSs can be achieved. Therefore, the RSs can reliably decode the signals with negligible errors [5]. In the $i^{\text {th }}$ sub-frame, $\mathrm{RS}_{\mathrm{i}}$ forwards $T_{i}$ symbols to UT using $M_{i}$-QAM modulation. The frame structure is depicted in Fig. 2.

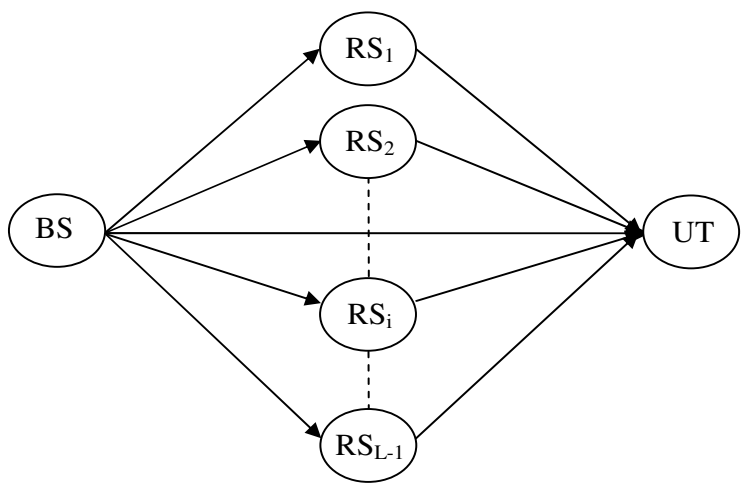

Figure 1. System Model
In the $i^{\text {th }}$ sub-frame and the $j^{\text {th }}$ symbol, the received signal at $\mathrm{UT}$ is $r_{i j}^{M_{i}}$ and given by $r_{i, j}^{M_{i}}=\alpha_{i} S_{i, j}^{M_{i}}+n_{i, j}$. The complex additive white Gaussian noise (AWGN) is represented by $n_{i, j}$, and it is modeled as $\operatorname{CN}\left(0, N_{0} / 2\right)$. The channel coefficient between the transmitting node $i$ and UT is denoted by $\alpha_{i}$ and it captures the effects of both path loss and small scale fading due to multipath propagation. Flat slow fading is considered, i.e. the channel does not change for the whole sub-frame. It is assumed that $\alpha_{i}$ 's are known at the receiver and modeled as independent $\operatorname{CN}\left(0, \sigma_{i}^{2}\right)$, with $\sigma_{i}^{2}=E\left\{\left|\alpha_{i}\right|^{2}\right\}$. If full channel state information (CSI) is available at BS, optimizing the modulation levels for all the transmitting nodes can improve the end to end throughput significantly. Such optimization is studied extensively in [5] and [6] and will not be repeated here. The instantaneous signal to noise ratio (SNR) of the link from node $i$ to UT is $\gamma_{i}=\left|\alpha_{i}\right|^{2} / N_{0}$ and the average SNR is $\bar{\gamma}_{i}=\sigma_{i}^{2} / N_{0}$.

After receiving all the sub-frames, UT can utilize the signals received from $L$ independent branches, and achieves spatial diversity.

Remarks:

1- For mathematical completeness, the most general case of $L-1$ relays is considered. However, given that each relay requires an orthogonal channel, it will be difficult in practice to have more than two relays due to the limited radio resources.

2- For simplicity, channel coding is not considered in this paper. However, the proposed scheme works equally well as long as all transmitting nodes use the same channel coding scheme.

\section{OPTIMAL DETECTOR}

\section{A. M-QAM modulation with Gray coding}

In this research, we focus on square M-QAM modulations with Gray coding, since these modulation schemes are the most popular schemes in wireless networks [2].

For a given sequence of bits $\left\{s_{1}, s_{2}, \ldots, s_{K}\right\}$, where $s_{i} \in\{-1,1\}$, we present a novel mathematical model that maps these $K$ bits into a Gray-coded M-QAM symbol, $S^{M}$. The model is given by,

$$
S^{M}\left(s_{1}, s_{2}, \ldots, s_{K}\right)=\left(-\chi_{K / 2}+j \beta_{K / 2}\right) d_{M}
$$

where $d_{M}$ is an arbitrary constant used to control the energy of the constellation. For convenience, the average energy per bit is fixed to unity by setting [7]:

$$
d_{M}=\sqrt{\frac{3 \log _{2} M}{2(M-1)}}
$$


The coefficients $\chi_{K / 2}$ and $\beta_{K / 2}$ can be computed recursively as follow:

$$
\begin{aligned}
& \chi_{k}=\left\{\begin{array}{cc}
-s_{K / 2} & k=1 \\
-s_{K / 2-k+1}\left(\chi_{k-1}+2^{k-1}\right) & 1<k \leq K / 2
\end{array}\right\} \\
& \beta_{k}=\left\{\begin{array}{cc}
-s_{K} & k=1 \\
-s_{K-k+1}\left(\beta_{k-1}+2^{k-1}\right) & 1<k \leq K / 2
\end{array}\right\}
\end{aligned}
$$

The same model derived above can be used to generate different Gray-coded constellations by either re-labeling the bits, or by negating the sign of the bits. The followings are examples of 4-QAM, 16-QAM, and 64-QAM Gray-coded symbols:

$$
\begin{gathered}
S^{4}\left(s_{1}, s_{2}\right)=s_{1} d_{4}-j s_{2} d_{4} \\
S^{16}\left(s_{1}, s_{2}, s_{3}, s_{4}\right)=s_{1}\left(-S_{2}+2\right) d_{16}-j s_{3}\left(-s_{4}+2\right) d_{16} \\
S^{64}\left(s_{1}, s_{2}, s_{3}, s_{4}, s_{5}, s_{6}\right)=s_{1}\left(-s_{2}\left(-s_{3}+2\right)+4\right) d_{64} \\
-j s_{4}\left(-s_{5}\left(-s_{6}+2\right)+4\right) d_{64}
\end{gathered}
$$

This model is essential for the MLD, which is described in the next section.

\section{B. Maximum Likelihood Detector (MLD)}

The optimum solution to the problem at hand is the MLD. For additive white Gaussian noise, the MLD reduces to a minimum distance classifier [7]. Consequently, the MLD decides on the sequence $\left[\hat{s}_{1}, \ldots, \hat{s}_{C}\right]$ that satisfies the following criterion:

$$
\begin{aligned}
& {\left[\hat{s}_{1}, \ldots, \hat{s}_{C}\right]=} \\
& \arg \min _{s_{1}, \ldots, s_{C}} \sum_{i=0}^{L-1} \sum_{j=0}^{T_{i}-1}\left|r_{i, j}^{M_{i}}-\alpha_{i} S_{i, j}^{M_{i}}\left(s_{j K_{i}+1}, s_{j K_{i}+2}, \ldots, s_{(j+1) K_{i}}\right)\right|^{2}
\end{aligned}
$$

where $S_{i, j}^{M_{i}}\left(s_{j K_{i}+1}, S_{j K_{i}+2}, \ldots, S_{(j+1) K_{i}}\right)$ is given by

Although the MLD achieves the optimum performance, it has exorbitant computational complexity. For example, to perform the decoding in the case where $M_{0}=4\left(K_{0}=2\right), M_{I}=16\left(K_{I}=4\right)$, and $M_{2}=64\left(K_{2}=6\right)$, the MLD decodes $C=\operatorname{LCM}(\{2,4,6\})=12$ bits jointly. This requires $2^{12}$ computations, which is clearly too complex. For this reason, we are motivated to provide practical schemes with much reduced complexity and comparable performance to the MLD. The proposed receiver schemes are described in the subsequent section.

\section{THE PROPOSED RECEIVER SCHEMES}

\section{A. Soft-Bit Maximum Likelihood Detector (SBMLD)}

Suboptimum schemes are proposed to overcome the complexity of the MLD. In the MLD, the complexity arises from the fact that different modulation schemes carry different number of bits per symbol. As a result, bit-by-bit (or symbolby-symbol) decoding is not possible. A trivial remedy to this problem is to decode the bits from each link and perform diversity combing on the hard bits. However, it is found out through simulation that this solution doesn't achieve full diversity because of the lost soft information in the hard decoding. In order to avoid losing the soft-bit information, we propose to map the received $M_{i}$-QAM soft-symbol, $r_{i, j}^{M_{i}}$, into $K_{i}$ soft-bits. Then, decoding can be preformed on the soft-bits, which is a bit-by-bit detection, rather than detecting a sequence of bits jointly.

To extract soft-bits from a soft-symbol, the logarithm of likelihood ratio (LLR) can be used. For Gray-coded $M_{i}$-QAM schemes described by (1), the LLR can be well approximated by the following recursive expression [8][9]:

$$
\tilde{s}_{i, j K_{i}+k}=\left\{\begin{array}{cc}
\operatorname{Re}\left\{\alpha_{i}^{*} r_{i, j}^{M_{i}}\right\} & k=1 \\
2^{\frac{K_{i}-k+1}{2}} d_{M_{i}}\left|\alpha_{i}\right|^{2}-\left|\tilde{s}_{i, j K_{i}+k-1}\right| & 1<k \leq \frac{K_{i}}{2} \\
-I m\left\{\alpha_{i}^{*} r_{i, j}^{M_{i}}\right\} & k=\frac{K_{i}}{2}+1 \\
2^{K_{i}-k+1} d_{M_{i}}\left|\alpha_{i}\right|^{2}-\left|\tilde{s}_{i, j K_{i}+k-1}\right| & \frac{K_{i}}{2}+1<k \leq K_{i}
\end{array}\right\}
$$

where $\tilde{s}_{i, j K_{i}+k}$ is the $\left(j K_{i}+k\right)^{\text {th }}$ soft-bit generated from the received soft-symbol $r_{i, j}^{M_{i}}$. Note that multiplying the received soft-symbol by the conjugate of the channel coefficient is necessary to undo the phase rotation caused by the channel.

The SBMLD, which is bit-by-bit detector, performs maximum likelihood detection on the soft-bits. It decides on the bit $\hat{s}_{l}$, for $l \in\{0,1, \ldots, C-1\}$, according to the following criterion:

$\left\{\begin{array}{cc}\hat{s}_{l}=1 & f_{\tilde{s}_{0, l}, \ldots, \tilde{s}_{L-1, l} \mid s_{l}=1}\left(\tilde{s}_{0, l}, \ldots, \tilde{s}_{L-1, l}\right)>f_{\tilde{s}_{0, l}, \ldots, \tilde{s}_{L-1, l} \mid s_{l}=-1}\left(\tilde{s}_{0, l}, \ldots, \tilde{s}_{L-1, l}\right) \\ \hat{s}_{l}=-1 & \text { otherwise }\end{array}\right\}$

Since the symbols received from different nodes are statistically independent, the joint conditional PDF is the multiplication of the marginal conditional PDFs. Hence, equation (6) reduces to:

$$
\left\{\begin{array}{c}
\hat{s}_{l}=1 \quad \text { if } \prod_{i=0}^{L-1} f_{\tilde{s}_{s_{l}} \mid s_{l}=1}\left(\tilde{s}_{i, l}\right)>\prod_{i=0}^{L-1} f_{\tilde{s}_{s_{l}} \mid s_{l}=-1}\left(\tilde{s}_{i, l}\right) \\
\hat{s}_{l}=-1
\end{array}\right\}
$$

From equation (6), it is easy to see that the marginal conditional PDFs of $\tilde{s}_{i, j K_{i}+k}$ is the same as that of $\tilde{s}_{i, k}$, for $j \in\left\{0,1, \ldots, T_{i}-1\right\}$. Thus, it suffices to provide the expression of the conditional PDFs of $\tilde{s}_{i, k}$ for $k \in\left\{1,2, \ldots, K_{i}\right\}$.

The conditional PDF of the soft-bits can be obtained from (6) by using the following properties that apply to any random variable $X$ and constant $c$ :

1) $f_{|X|}(x)=\left\{\begin{array}{cc}f_{X}(x)+f_{X}(-x) & x \geq 0 \\ 0 & x<0\end{array}\right.$

2) $f_{X+c}(x)=f_{X}(x-c)$

The conditional PDFs of the soft-bits are given by:

$$
\begin{aligned}
& f_{\tilde{s}_{i, k} \mid s_{n}= \pm 1}(x)= \\
& \left\{\begin{array}{cc}
\frac{2}{K_{i}} \sum_{m=1}^{K_{i} / 2} g\left(x ; \mu_{n, m}^{ \pm 1}\left|\alpha_{i}\right|^{2} d_{M_{i}}\left|\alpha_{i}\right|^{2} N_{0} / 2\right) & k=1 \\
f_{\tilde{s}_{i, k-1} \mid s_{n}= \pm 1}\left(x-\eta_{i, k}\right)+f_{\tilde{s}_{i, k-1} \mid s_{n}= \pm 1}\left(-x+\eta_{i, k}\right) & 1<k \leq \frac{K_{i}}{2}, x \leq \eta_{i, k} \\
0 & 1<k \leq \frac{K_{i}}{2}, x>\eta_{i, k} \\
f_{\tilde{s}_{i, k-K_{i} \mid} \mid s_{n}= \pm 1}(x) & \frac{K_{i}}{2}<k \leq K_{i}
\end{array}\right.
\end{aligned}
$$


where $\eta_{i, k}=2^{\frac{K_{i}}{2}-k+1} d_{M_{i}}\left|\alpha_{i}\right|^{2}$ and $n \in\left\{1,2, \ldots, K_{i}\right\}$. The elements of the matrices $\boldsymbol{\mu}^{+1}$ and $\boldsymbol{\mu}^{-1}$ are tabulated in Table I for the modulation schemes described by (4). Note that the detection rule in this case didn't simplify to a minimum distance classifier, as in the case of the MLD, because the conditional PDFs of the soft-bits are not Gaussian.

\section{B. Soft-Bit Maximum Ratio Combiner (SBMRC)}

To avoid the computation of the conditional PDF's, and have a scheme that has complexity comparable to the classical MRC, we propose SBMRC.

The SBMRC weights the soft-bits according to their reliabilities and adds them in a way similar to MRC, hence the name SBMRC. The difference in reliabilities comes as a result of two factors. First, the soft-symbols (consequently the softbits) experience different channel conditions. Since the softbits are already weighted according to their channel conditions by the definition in (6), there is no need to weight them again in the combining. Second, the reliabilities of the soft-bits vary according to their modulation levels. Thus, we propose to weight the soft-bits with $d_{M_{i}}$ which is given by (2). The rational behind choosing $d_{M_{i}}$ in such a manner is to scale the soft-bits that are generated from denser constellations with less weight, as they are more vulnerable to noise, and vice versa.

Consequently, the SBMRC decides on the bit $\hat{s}_{l}$, for

$l \in\{1,2, \ldots, C\}$, according to the following criterion:

$$
\begin{cases}\hat{s}_{l}=1 & \text { if } \sum_{i=0}^{L-1} d_{M_{i}} \tilde{s}_{i, l}>0 \\ \hat{s}_{l}=-1 \quad & \text { otherwise. }\end{cases}
$$

\section{Simulation Results}

In this section, we present simulation results of our schemes. The conditional PDFs for the soft-bits generated from a soft 16-QAM symbol (i.e. $M_{i}=16$ ) are shown in Fig. 3 and Fig. 4. In both cases, the theoretical conditional PDFs given by (9) are in good agreement with the simulation results, and it is evident that the conditional PDFs don't follow a Gaussian distribution for low SNR.

To compare the performance of SBMLD, SBMRC, and MLD, we consider relay networks with $L=2$ (single relay) and $L=3$ (two relays). For simplicity, we assume the average channel conditions to be the same in all the links, i.e., $\bar{\gamma}_{i}=\bar{\gamma}$ for $i \in\{0,1, \ldots, L-1\}$. Table II shows the loss in SNR of the SBMLD and SBMRC schemes compared to the optimum MLD scheme for different scenarios. It is clear that both SBMLD and SBMRC have very close performance to the MLD scheme (degradation is less than $0.3 \mathrm{~dB}$ ). The loss in SNR was measured at a BER of $10^{-3}$ which is a reasonable value for uncoded schemes. Nevertheless, it was observed that the loss in SNR vanishes at very low BER. The SBMLD provides negligible performance gain compared to the SBMRC at the expense of the computation of the conditional PDFs of the soft-bits. For these reasons, throughout this discussion, we will focus on SBMRC, since it is very simple to implement, with negligible degradation compared to the MLD and SBMLD schemes.

Fig. 5 and Fig. 6 show the BER performance of SBMRC for combining signals with different modulation schemes, for single and two relays, respectively. It is clear that all curves in Fig. 5 decay 2 orders of magnitude per decade (diversity order of 2) and all curves in Fig. 6 decay 3 orders of magnitude per decade (diversity order of 3 ). Consequently, SBMRC achieves full diversity (diversity order of $L$ ). Note that the results for the case where $M_{i}=2$ (BPSK) were not shown since both BPSK and QPSK have the same BER performance.

In Fig. 7, we compare the BER performance of SBMRC with the conventional selection combining, in a single relay network $(L=2)$. We consider the cases where the average SNR in the BS-UT link $\left(\bar{\gamma}_{0}\right)$ is less than the average SNR in the $\mathrm{RS}_{1}$-UT link $\left(\bar{\gamma}_{1}\right)$ by 5,10 , and $15 \mathrm{~dB}$. This represents practical scenarios where UT is closer to $\mathrm{RS}_{1}$ than BS. For all cases, our scheme outperforms conventional selection combining by almost $2 \mathrm{~dB}$. For the sake of presentation, we use the case where $M_{0}=4$ and $M_{I}=64$; however, the same gain has been observed for different set of modulations. It is interesting to note that this is close to the gain that classical MRC provides over selection combining, in the case of combining signals with the same modulation levels.

\section{CONCLUSIONS}

In the current literature, diversity combining of signals with different modulation levels in cooperative relay networks has been addressed by means of selection combining, which is far from optimal. In this paper, we have proposed optimal as well as near-optimal and low complexity diversity combining schemes of signals with different modulation levels. The optimum detector is developed as an MLD detector. To overcome the complexity of the MLD, we have proposed SBMLD and SBMRC, which are simple bit-by-bit detectors and are only $0.3 \mathrm{~dB}$ inferior to the optimal MLD in performance. The SBMLD provides only marginal performance gain over SBMRC through the computation of the conditional PDFs of the soft-bits. Consequently, the SBMRC is a more attractive practical solution. The SBMRC, along with its simplicity, outperforms selection combining by almost $2 \mathrm{~dB}$ without any bandwidth loss and without the need for extra channel state information. The proposed scheme can be viewed as a more general form of the classical MRC and it is an essential scheme to incorporate cooperative diversity with AMC in next generation wireless networks.

\section{REFERENCES}

[1] B. H. Walke, R. Pabst, D. Schultz, P. Herhold, H. Yanikomeroglu, S. Mukherjee, H. Viswanathan, M. Lott, W. Zirwas, M. Dohler, H. Aghvami, D. D. Falconer, and G. P. Fettweis, "Relay-based deployment concepts for wireless and mobile broadband radio," IEEE Commun. Mag., vol. 42, no. 9, pp. 80-89, Sep. 2004.

[2] Wimax Forum, "Mobile WiMAX- Part I: A Technical Overview and Performance Evaluation," June 2006. 
[3] N. Laneman, D. Tse, and G. Wornell, "Cooperative diversity in wireless networks: Efficient protocols and outage behavior," IEEE Trans. On Information Theory, vol. 50, pp. 3062-3080, Dec. 2004.

[4] J. Boyer, D. D. Falconer, and H. Yanikomeroglu, "Multihop diversity in wireless relaying channels," IEEE Trans. Commun., vol. 52, no. 10, pp. 1820-1830, Oct. 2004.

[5] B. Can, H. Yanikomeroglu, F. Onat, E. Carvalho and H. Yomo, "Efficient Cooperative Diversity Schemes and Radio Resource Allocation for IEEE 802.16j," IEEE WCNC 2008, 31 March - 3 April 2008, Las Vegas, USA.

[6] S. Hares, H. Yanikomeroglu, and B. Hashem, "Diversity- and AMC (adaptive modulation and coding)-aware routing in TDMA peer-to-peer multihop networks," IEEE GLOBECOM 2003, 1-5 December 2003, San Francisco, CA, USA.

[7] J. G. Proakis, Digital Coomunications. McGraw-Hill, 2000.

[8] S. Le Goff, A. Glavieux, and C. Berrou, "Turbo-codes and high spectral efficiency modulation,” Proc. ICC'94 New Orleans, LA, May 1994.

[9] V. Aue and R. Nuessgen. "Method for generating soft-bit information from Gray coded signals.” U.S. Patent 20040096007, May. 20, 2004.

Table I. The matrices $\boldsymbol{\mu}^{+1}$ and $\boldsymbol{\mu}^{-1}$ for different M-QAM constellations.

\begin{tabular}{|c|c|c|}
\hline $\begin{array}{c}\text { Modulation } \\
\text { Level }\left(\mathrm{M}_{\mathrm{i}}\right)\end{array}$ & $\boldsymbol{\mu}^{+1}$ & $\boldsymbol{\mu}^{-1}$ \\
\hline 4 & $\boldsymbol{\mu}^{+1}=1$ & $\boldsymbol{\mu}^{-1}=-1$ \\
\hline 16 & $\boldsymbol{\mu}^{+1}=\left[\begin{array}{cc}1 & 3 \\
-1 & 1\end{array}\right]$ & $\boldsymbol{\mu}^{-1}=\left[\begin{array}{cc}-1 & -3 \\
-3 & 3\end{array}\right]$ \\
\hline 64 & $\boldsymbol{\mu}^{+1}=\left[\begin{array}{cccc}1 & 3 & 5 & 7 \\
-1 & 1 & -3 & 3 \\
-3 & 3 & -5 & 5\end{array}\right]$ & $\boldsymbol{\mu}^{-1}=\left[\begin{array}{cccc}-1 & -3 & -5 & -7 \\
-5 & 5 & -7 & 7 \\
-1 & 1 & -7 & 7\end{array}\right]$ \\
\hline
\end{tabular}

Table II. Loss in SNR (dB) at BER $=10^{-3}$ of SBMLD and SBMRC compared to the optimum MLD.

\begin{tabular}{|c|c|c|}
\hline Scenario & SBMLD & SBMRC \\
\hline$M_{0}=4, M_{1}=16$ & 0.001 & 0.020 \\
\hline$M_{0}=4, M_{1}=64$ & 0.056 & 0.090 \\
\hline$M_{0}=16, M_{1}=64$ & 0.062 & 0.081 \\
\hline$M_{0}=4, M_{1}=4, M_{2}=16$ & 0.040 & 0.069 \\
\hline$M_{0}=4, M_{1}=4, M_{2}=64$ & 0.221 & 0.273 \\
\hline$M_{0}=4, M_{1}=16, M_{2}=16$ & 0.087 & 0.095 \\
\hline$M_{0}=16, M_{1}=16, M_{2}=64$ & 0.120 & 0.135 \\
\hline$M_{0}=16, M_{1}=64, M_{2}=64$ & 0.092 & 0.092 \\
\hline
\end{tabular}

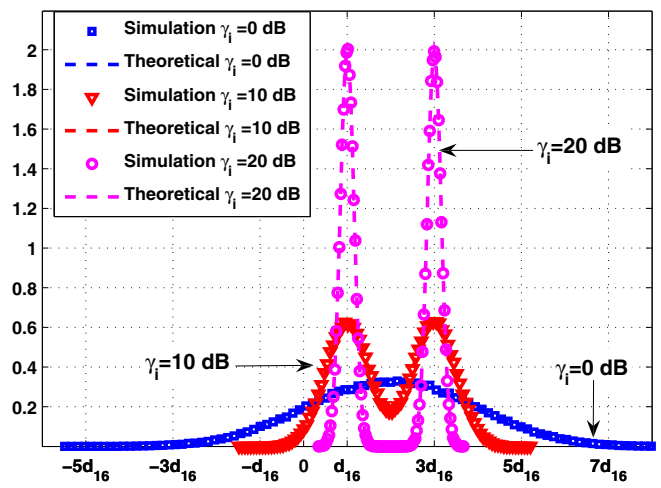

Figure 3. Conditional PDF of $\tilde{s}_{i, 1}\left(\tilde{s}_{i, 3}\right)$ generated from a 16-QAM symbol, given that $s_{1}=1 \quad\left(s_{3}=1\right)$, for different channel conditions.

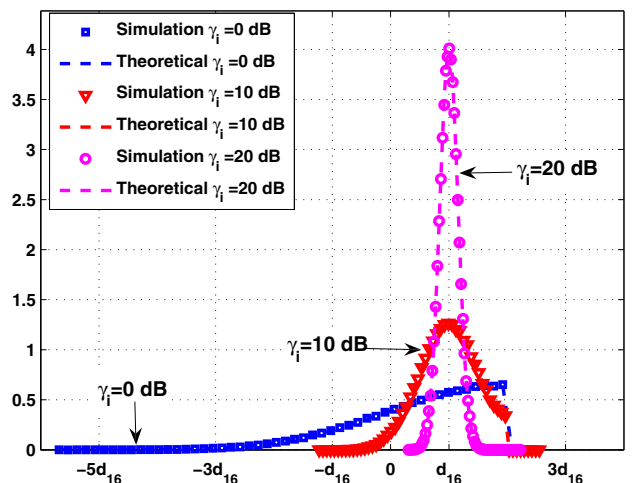

Figure 4. Conditional PDF of $\tilde{s}_{i, 2}\left(\tilde{s}_{i, 4}\right)$ generated from a 16-QAM symbol, given that $s_{2}=1 \quad\left(s_{4}=1\right)$, for different channel conditions.

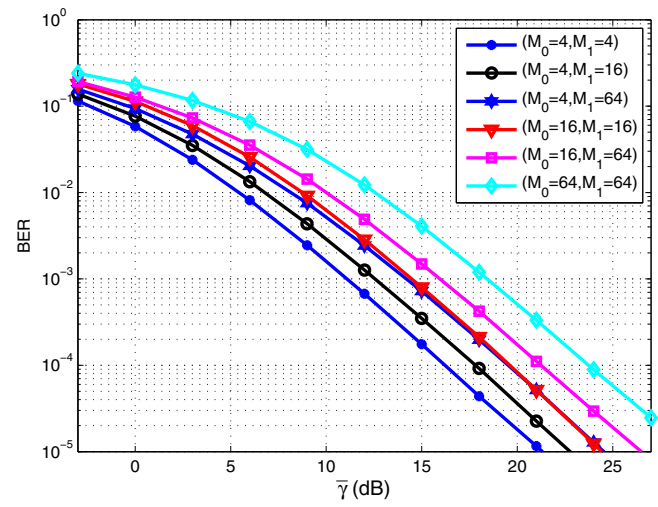

Figure 5. BER performance of diversity combining using SBMRC, for $L=2$.

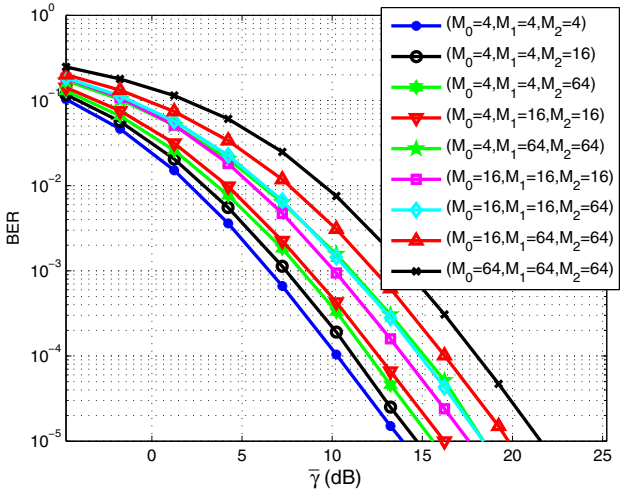

Figure 6. BER performance of diversity combining using SBMRC, for $L=3$.

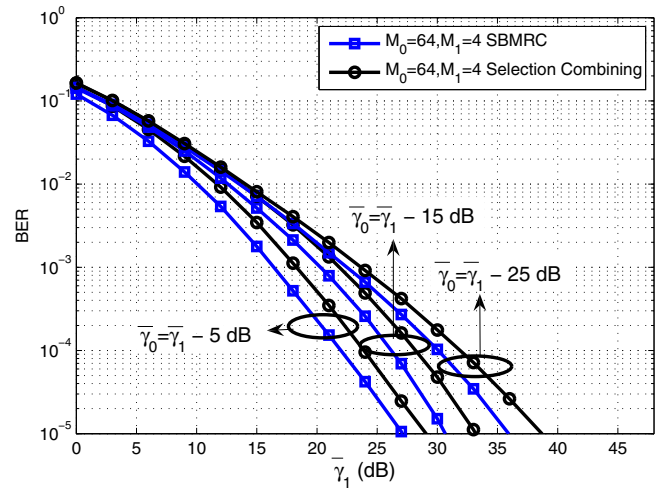

Figure 7. BER performance comparison of Selection Combining with SBMRC. 